\title{
The Theory of Pure Algebraic (Co)Homology
}

\author{
Alaa Hassan Noreldeen ${ }^{1, *}$, Wageeda M. M. ${ }^{1}$, O. H. Fathy ${ }^{2}$ \\ ${ }^{1}$ Department of Mathematics, Faculty of Science, Aswan University, Aswan, Egypt \\ ${ }^{2}$ Department of Basic and Applied Sciences, Arab Academy for Science, Technology \& Maritime Transport, Aswan, Egypt
}

Received June 8, 2021; Revised July 12, 2021; Accepted August 22, 2021

\section{Cite This Paper in the following Citation Styles}

(a): [1] Alaa Hassan Noreldeen, Wageeda M. M., O. H. Fathy , "The Theory of Pure Algebraic (Co)Homology," Mathematics and Statistics, Vol. 9, No. 5, pp. 639 - 647, 2021. DOI: 10.13189/ms.2021.090503.

(b): Alaa Hassan Noreldeen, Wageeda M. M., O. H. Fathy (2021). The Theory of Pure Algebraic (Co)Homology. Mathematics and Statistics, 9(5), 639 - 647. DOI: 10.13189/ms.2021.090503.

Copyright@2021 by authors, all rights reserved. Authors agree that this article remains permanently open access under the terms of the Creative Commons Attribution License 4.0 International License

\begin{abstract}
Polynomial: algebra is essential in commutative algebra since it can serve as a fundamental model for differentiation. For module differentials and Loday's differential commutative graded algebra, simplified homology for polynomial algebra was defined. In this article, the definitions of the simplicial, the cyclic, and the dihedral homology of pure algebra are presented. The definition of the simplicial and the cyclic homology is presented in the Algebra of Polynomials and Laurent's Polynomials. The long exact sequence of both cyclic homology and simplicial homology is presented. The Morita invariance property of cyclic homology was submitted. The relationship $\mathcal{H} C_{n}(\mathcal{P}) \cong{ }^{\alpha} \mathcal{H} \mathcal{D}_{n}(\mathcal{P}) \oplus$ $-\alpha \mathcal{H} \mathcal{D}_{n}(\mathcal{P}), \alpha= \pm 1$ was introduced, representing the relationship between dihedral and cyclic (co)homology in polynomial algebra. Besides, a relationship $\mathcal{H} C_{n}\left(\mathcal{P}\left[p, p^{-1}\right]\right) \cong$

${ }^{\alpha} \mathcal{H} \mathcal{D}_{n}\left(\mathcal{P}\left[\not p, p^{-1}\right]\right) \oplus^{-\alpha} \mathcal{H} \mathcal{D}_{n}\left(\mathcal{P}\left[\not p, p^{-1}\right]\right) \quad, \quad \alpha= \pm 1$ was examined, defining the relationship between dihedral and cyclic (co)homology of Laurent polynomials algebra. Furthermore, the Morita invariance property of dihedral homology in polynomial algebra was investigated. Also, the Morita property of dihedral homology in Laurent polynomials was studied. For the dihedral homology, the long exact sequence $\cdots \rightarrow \mathcal{H} \mathcal{D}_{n}(\mathcal{P}) \rightarrow \mathcal{H} \mathcal{D}_{n}\left(\mathcal{P}^{\prime}\right) \rightarrow$ $\mathcal{H} \mathcal{D}_{n}\left(\mathcal{P}^{\prime \prime}\right) \rightarrow \mathcal{H} \mathcal{D}_{n-1}(\mathcal{P}) \rightarrow \mathcal{H} \mathcal{D}_{n-1}\left(\mathcal{P}^{\prime}\right) \rightarrow$ $\mathcal{H} \mathcal{D}_{n-1}\left(\mathcal{P}^{\prime \prime}\right) \rightarrow \cdots$ was obtained of the short sequence $0 \rightarrow \mathcal{P} \rightarrow \mathcal{P}^{\prime} \rightarrow \mathcal{P}^{\prime \prime} \rightarrow 0$. The long exact sequence of the short sequence $0 \rightarrow \mathcal{P} \rightarrow \mathcal{P}^{\prime} \rightarrow \mathcal{P}^{\prime \prime} \rightarrow 0$ was obtained from the reflexive (co)homology of polynomial algebra. Studying polynomial algebra helps calculate COVID-19 vaccines.
\end{abstract}

Keywords Homology Theory, Pure Algebras, Exact Sequence, Polynomial Algebra, Dihedral Homology
2010 Mathematics Subject Classifications: 55N35

\section{Introduction}

The homology theory of algebras over a field refers to the Hochschild (co)homology in the mathematical field. Hochschild introduced simplicial cohomology for algebras [1], and Henri and Samuel expanded it over rings in [2].

Certain (co)homology theories for associative algebras in disciplines of mathematics and non-commutative geometry that generalise de Rham homology and cohomology of manifolds are referred to as cyclic (co)homology. Boris Tsygan [3] and Alain Connes [4] pioneered the concepts of homology and cohomology independently. Many older branches of mathematics, such as de Rham's speculation, simplicial (co)homology, group (co)homology, and $\mathcal{K}$-theory, have intriguing relationships with these invariants.

The dihedral (co)homology, proposed independently and demonstrated in various algebras, is the hermitian equivalent of a cyclic (co)homology [4]. The dihedral homology of algebras over the field is defined as the algebraic homology of the dihedral group [5]. The dihedral (co)homology is denoted as (co)homology with group symmetry [6]. There are two types of (co)homology theory: in-discrete and discrete. The Hochschild mentioned in reference [1] is related to Hochschild in the discrete field's (co)homology of algebra with $i d$. The first nontrivial (co)homology group was introduced by Tsygan [3] and Connes [4]. In 1987, the involutive unital 
algebra's reflexive and dihedral (co)homology was investigated, and the remaining (co)homology group was studied in 1989. The analogue simplified cohomology of operator algebras was studied [7]. The Banach cyclic (co)homology has been explored [8] [9] [10]. The group Banach dihedral cohomology and its relation to cyclic cohomology were investigated [12]. The dihedral cohomology groups of certain operator algebras were analysed [6]. Calculating operator algebras' group symmetry, bisymmetry, and Weil (co)homology have stalled, but the cohomology module $\mathcal{K}$-module was investigated [13].

Grothendieck made a breakthrough in cohomology with his proof of the Zeta function $Z_{x}(t)$ of finite scheme over finite ring $F_{q}$. He proved that the factor $\mathcal{P}_{i}(t)$ of $Z_{x}(t)$ be a polynomial that operates on a $l$-adic cohomology group, namely $H^{i}\left(\mathcal{X}, \mathbb{Q}_{l}\right)=\lim _{\rightarrow} H_{e t}^{i}(\mathcal{X}, \mathbb{Z} /$ $\left.\left(l^{v}\right)\right)$.

Deligne proved the "Riemann hypothesis" over $F_{q_{\text {, }}}$ using étale cohomology in 1972: the Frobenius eigenvalues on $H^{i}\left(\mathcal{X}, \mathbb{Q}_{l}\right)$ were correct algebraic numbers with absolute value $q^{i / 2}$. It was the final step in proving the famous Weil Conjectures, cementing the value of étale cohomology. Bernstein, Gelfand, and Gelfand classified vector bundles on projective space $\mathcal{P}^{n}$ over a field $\mathcal{K}$ in 1978 using derived categories as graded modules over the exterior algebra $\mathcal{A}$ on $n+1$ variables. The discovery of an isomorphism between the bounded derived categories of graded modules $\mathcal{D}_{g r}^{b}(\mathcal{R})$, and $\mathcal{D}_{g r}^{b}(\mathcal{A})$, where polynomial algebra on $n+1$ variables is $\mathcal{R}$, was a crucial step in classifying them. This result revealed that $\mathcal{D}^{b}(\mathcal{A})$ did not evaluate the category $W$, which was unexpected (see [14], [15]).

In 1985 and 1987, cyclic homology of algebra was determined when the characteristic is 0 . In 1991, if $\mathcal{F}$ is polynomial and $\mathcal{K}$ is a ring with unital, the cyclic homology of algebra $\mathcal{A}=\mathcal{K}[X] /(\mathcal{F})$ is calculated. The dimensions of simplicial (co)homology of periodic infinite algebras of polynomial growth were defined [11]. For applying this, a nonstandard periodic representation was obtained that is not analysable since the polynomial's infinite algebra does not derive from scalar algebra.

The first section will outline the definitions of Hochschild homology and cyclic homology. The long exact sequences of simplicial homology and cyclic homology will be presented. In the second part, the algebraic definitions of polynomials and Laurentian polynomials will be reviewed. The simplicial and cyclic homology will be identified first, followed by a discussion of their leng exact sequences. In part three, the dihedral homology of polynomial algebra will be examined, discussing the long exact between cyclic, dihedral homology in polynomial algebra and Laurent polynomials algebra. The Morita of dihedral homology property and Morita's invariance for dihedral homology will be examined in polynomial algebra and Laurent polynomials algebra, implying that the trace map is inverse to the inclusion map.

The following section contains definitions for the homology theorem. A Hochschild homology description of pure algebra will be presented. The concept of cyclic homology will be expanded, defining both reflexive and dihedral algebraic homology.

\section{Homology Theory of Algebras}

The cyclic and dihedral homologies for pure algebra were introduced to define Hochschild homology. Let $\mathcal{A}$ be a pure algebra over $\mathcal{K}$ and $\mathcal{M}$ bimodule with involution $\quad *: \mathcal{A} \rightarrow \mathcal{A} ; a \rightarrow a^{*} \forall a \in \mathcal{A}$. First, the Hochschild homology was determined by defining the chain complex

$$
\mathrm{C}(\mathcal{A}):=\cdots \rightarrow \mathcal{A}_{0} \stackrel{d_{0}}{\rightarrow} \mathcal{A}_{1} \stackrel{d_{1}}{\rightarrow} \mathcal{A}_{2} \stackrel{d_{2}}{\rightarrow} \cdots
$$

using the operator

$$
\begin{gathered}
d_{n}\left(a_{0}, a_{1}, \ldots, a_{n}\right)= \\
\sum_{\mathrm{i}=0}^{n-1}(-1)^{\mathrm{i}}\left(a_{0}, \ldots, a_{\mathrm{i}} \cdot a_{\mathrm{i}+1}, \ldots, a_{n}\right)+ \\
(-1)^{n}\left(a_{n} . a_{0}, a_{1}, \ldots, a_{n-1}\right),
\end{gathered}
$$

where $d^{2}=d_{n} d_{n+1}=0$.

$$
\begin{gathered}
\text { If } Z\left(C_{*}(\mathcal{A})\right)=\operatorname{ker}\left(d_{n}\right), \mathcal{B}\left(C_{*}(\mathcal{A})\right)=\operatorname{Im}\left(d_{n+1}\right) \\
\text { and } \operatorname{Im}\left(d_{n+1}\right) \subset \operatorname{ker}\left(d_{n}\right),
\end{gathered}
$$

then the (co)homology of the upper complex is

$$
\mathcal{H}_{n}\left(C_{*}(\mathcal{A})\right)=\frac{z\left(C_{*}(\mathcal{A})\right)}{\mathcal{B}\left(C_{*}(\mathcal{A})\right)}=\frac{\operatorname{ker}\left(d_{n}\right)}{\operatorname{Im}\left(d_{n+1}\right)} .
$$

It is defined as $\mathcal{A}$-simplicial algebra's homology and is denoted by $\mathcal{H H}_{n}(\mathcal{A})$. Another definition of Hochschild (co)homology was reported [16]. If $\mathcal{A}$ is a tensor product $\mathcal{A}^{e}=\mathcal{A} \otimes \mathcal{A}^{o p}$, it can be defined by (Tor) and (Ext) as following:

$$
\begin{gathered}
\mathcal{H H}_{n}(\mathcal{A}, \mathcal{M})=\operatorname{Tor}_{n}^{\mathcal{A}}(\mathcal{A}, \mathcal{M}), \\
\mathcal{H H}^{n}(\mathcal{A}, \mathcal{M})=\operatorname{Ext}_{\mathcal{A}}^{n}(\mathcal{A}, \mathcal{M}) .
\end{gathered}
$$

Before defining periodic homology, the cyclic operator $t_{n}: C_{n}(\mathcal{A}) \rightarrow C_{n}(\mathcal{A})$ must be defined, where

$$
t_{n}\left(a_{0}, \ldots, a_{n-1}, a_{n}\right)=(-1)^{n}\left(a_{n}, a_{0}, \ldots, a_{n-1}\right) \text {. }
$$

If the next complex called the subcomplex of $C_{n}(\mathcal{A})$,

$$
\begin{array}{rrrrrrrrr}
\vdots & & \vdots & & \vdots & \vdots & & \\
\downarrow & & \downarrow & & \downarrow & \downarrow & & \\
C_{2} & 1-t & C_{2} & N & C_{2} & \stackrel{1-t}{\leftarrow} C_{2} & \stackrel{N}{\leftarrow} & \ldots \\
\mathrm{CC}_{n}(\mathcal{A}): b \downarrow & & -b^{\prime} \downarrow & b \downarrow & -b^{\prime} \downarrow & & \\
\mathrm{C}_{1} & \stackrel{1-t}{\leftarrow} & \mathrm{C}_{1} & N & \mathrm{C}_{1} & \stackrel{1-t}{\leftarrow} \mathrm{C}_{1} & \stackrel{N}{\leftarrow} & \ldots \\
b \downarrow & & -b^{\prime} \downarrow & b \downarrow & -b^{\prime} \downarrow & & \\
\mathrm{C}_{0} & \stackrel{1-t}{\leftarrow} & \mathrm{C}_{0} & \stackrel{N}{\leftarrow} & \mathrm{C}_{0} & \stackrel{1-t}{\leftarrow} \mathrm{C}_{0} & \stackrel{N}{\leftarrow} & \cdots
\end{array}
$$

where $\quad b=\sum_{i=0}^{n}(-1)^{i} d_{i}, b^{\prime}=\sum_{i=0}^{n-1}(-1)^{i} d_{i}, \quad$ and $N:=1+t+\cdots t^{n}$, then its homology called cyclic homology and given as follows ([17], [18]): 
$\mathcal{H C}_{n}(\mathcal{A})=\mathcal{H}_{n}\left(\mathrm{CC}_{*}(\mathcal{A}), b_{*}\right)=\mathcal{H}_{n}\left(\frac{\mathrm{C}_{n}(\mathcal{A})}{\operatorname{Im}\left(1-t_{n}\right)}, b_{*}\right)$

Alternatively, if $\Lambda$ is a category, the cyclic homology and cohomology are as follows:

$$
\begin{aligned}
\mathcal{H C}_{n}(\mathcal{M}) & =\operatorname{Tor}_{n}^{\mathcal{K}[\Lambda]^{o p}}\left(\mathcal{K}^{\mathrm{C}}, \mathcal{M}\right), \mathcal{H} \mathrm{C}^{n}(\mathcal{M}) \\
& =\operatorname{Ext}_{\mathcal{K}[\Lambda]^{o p}}^{n}\left(\mathcal{M}, \mathcal{K}^{\mathrm{C}}\right), n \geq 0 .
\end{aligned}
$$

The reflexive homology was defined by identifying a subcomplex of $\mathrm{C}_{n}(\mathcal{A})$ as following:

$$
{ }^{\alpha} \mathcal{R}_{n}(\mathcal{A})=\frac{\mathrm{C}_{n}(\mathcal{A})}{\operatorname{Im}\left(1-r_{n}\right)},
$$

with reflexive operator $r_{n}: \mathcal{C}_{n}(\mathcal{A}) \rightarrow \mathcal{C}_{n}(\mathcal{A})$, since

$r_{n}\left(a_{0}, \ldots, a_{n-1}, a_{n}\right)=a(-1)^{n(n+1) / 2}\left(a_{0}^{*}, a_{n}^{*}, \ldots, a_{1}^{*}\right)$.

Its (co)homology is given by

$\alpha \mathcal{H}_{\mathcal{R}_{n}}(\mathcal{A})=\mathcal{H}_{n}\left(\mathcal{C}_{*}(\mathcal{A}), d_{*}\right)=\mathcal{H}_{n}\left(\frac{C_{*}(\mathcal{A})}{\operatorname{Im}\left(1-r_{n}\right)}, d_{*}\right) \quad$, and it is called hyperhomology (hypercohomolog) of $\mathcal{A}$ [19].

The ${ }^{\alpha} C \mathcal{D}_{n}(\mathcal{A})=\left(\frac{C_{n}(\mathcal{A})}{\operatorname{Im}\left(1-t_{n}\right)+\operatorname{Im}\left(1-r_{n}\right)}\right)$ was obtained by using the (1) and (2) on $C(\mathcal{A})$, which is a sub-complex of $C_{n}(\mathcal{A})$. If the homology of this complex ${ }^{\alpha} \mathcal{C D}_{*}(\mathcal{A})$ is taken, the dihedral homology of algebra $\mathcal{A}$ can be defined. It is given by

$$
\begin{gathered}
\alpha \mathcal{H D}_{n}(\mathcal{A})=\mathcal{H}_{n}\left(\mathrm{CD}_{*}(\mathcal{A}), b_{*}\right)= \\
\mathcal{H}_{n}\left(\frac{C_{n}(\mathcal{A})}{\operatorname{Im}\left(1-t_{n}\right)+\operatorname{Im}\left(1-r_{n}\right)}, b_{*}\right) .
\end{gathered}
$$

An alternative perspective on dihedral (co)homology is as follows:

$$
\begin{gathered}
\operatorname{Tor}_{n}^{\mathcal{K}[\xi]^{o p}}\left(\mathcal{K}^{\mathcal{D}}, \mathcal{M}\right)=\mathcal{H} \mathcal{D}_{n}(\mathcal{M}), \\
\operatorname{Ext}_{\mathcal{K}[\xi]]^{o p}}^{n}\left(\mathcal{M}, \mathcal{K}^{\mathcal{D}}\right)=\mathcal{H} \mathcal{D}^{n}(\mathcal{M}), \quad n \geq 0 .
\end{gathered}
$$

In the following section, the basic definitions of a polynomial will be discussed. We present both the Hochschild homology and the cyclic homology definitions of the polynomial. The Morita Invariance is introduced for simplicial and cyclic homology more detail see ([19],[20],[21]).

\section{Homology of Polynomial Algebras}

Define $\mathcal{P}$ as algebra and $[\mathcal{P}]$ as class of polynomials;

$$
\mathcal{K}[\mathcal{P}]=\sum_{i=0}^{n} a_{i} p^{i}=\left(a_{0}, a_{1} p, a_{2} p^{2}, \ldots, a_{n} p^{n}\right),
$$

where $\mathcal{K}[p]$ is a polynomial algebra with the multiplication of coefficient of polynomials. A Laurent polynomial in a field $\mathcal{K}\left[\not p, p^{-1}\right]$ with coefficients is defined as follows:

$$
\mathcal{P}=\sum_{i} a_{i} \mathcal{P}^{i}, \quad \forall a_{i} \in \mathcal{K}\left[p, p^{-1}\right] .
$$

If the coefficients of two Laurent polynomials are the same, they are equal, achieving

$$
\left(\sum_{i} a_{i} x^{i}\right)+\left(\sum_{i} b_{i} x^{i}\right)=\sum_{i}\left(a_{i}+b_{i}\right) x^{i},
$$

and

$$
\left(\sum_{i} a_{i} x^{i}\right) \cdot\left(\sum_{j} b_{j} x^{j}\right)=\sum_{n}\left(\sum_{i, j, i+j=n} a_{i} b_{j}\right) x^{n} .
$$

Suppose that $\mathcal{P}[p]$ is a polynomial algebra over the $\mathcal{K}$ ring with an involution $*: \mathcal{P} \rightarrow \mathcal{P} ; p \rightarrow p^{*}$ for all $p \in \mathcal{P}$ [12]. We define a complex $\mathrm{C}(\mathcal{P}[\mathfrak{p}])=\left(\mathrm{C}_{n}(\mathcal{P}), b_{n}\right)$, since

$$
C_{n}(\mathcal{P})=\mathcal{P}^{\otimes(n+1)}, \quad b_{n} ; C_{n}(\mathcal{P}) \rightarrow C_{n-1}(\mathcal{P}), n \geq 0
$$

is the boundary operator

$$
\begin{aligned}
& b_{n}\left(p_{0}, p_{1}, \ldots, p_{n}\right) \\
= & \sum_{i=0}^{n-1}(-1)^{i}\left(p_{0}, \ldots, p_{i} p_{i+1}, \ldots, p_{n}\right) \\
+ & (-1)^{n}\left(p_{n} p_{0}, p_{1}, \ldots, p_{n-1}\right) .
\end{aligned}
$$

It is well known that $b_{n} b_{n+1}=0$. The simplicial homology of algebra $\mathcal{P}$, denoted by $\mathcal{H H}_{n}(\mathcal{P})$, is the homology of this complex;

$$
\mathcal{H}_{n}(\mathcal{P}[\not p])=\mathcal{H}_{n}\left(\mathcal{C}_{*}(\mathcal{P})\right)=\mathcal{H}_{n}(\mathcal{P}[\not p]) \otimes \mathcal{H}_{n}(\mathcal{K}[\not p]) .
$$

Since $\mathcal{P}\left[p, p^{-1}\right]=\bigotimes_{\mathcal{K}} \mathcal{K}\left[\not p, p^{-1}\right] \quad$ is Laurent polynomial algebra over the filed $\mathcal{K}\left[p, p^{-1}\right]$, the simplicial homology of $\mathcal{P}\left[p, p^{-1}\right]$ algebra is

$\mathcal{H H}_{n}\left(\mathcal{P}\left[\not p, p^{-1}\right]\right)=\mathcal{H} \mathcal{H}_{n}(\mathcal{P}[\not p]) \otimes \mathcal{H} \mathcal{H}_{n}\left(\mathcal{K}\left[\not p, p^{-1}\right]\right)$.

Concerning $\mathcal{P}[p]$ polynomial algebra, the cyclic homology of this is given by

$$
\mathcal{H} C_{n}(\mathcal{P}[p])=\mathcal{H} C_{n}(\mathcal{P}) \oplus \mathcal{H} \mathcal{H}_{n}^{\infty}(\mathcal{P}) .
$$

The cyclic homology of Laurent polynomial algebra $\mathcal{P}\left[\not p, p^{-1}\right]$ is

$$
\begin{array}{rl}
\mathcal{H} C_{n}\left(\mathcal{K}\left[p, p^{-1}\right]\right) & =\mathcal{K}[u] \otimes(\mathcal{K} .1 \oplus \mathcal{K} . d g) \oplus(1- \\
g & \mathcal{K}\left[p, p^{-1}\right] .
\end{array}
$$

If $k$ contains $\mathbb{Q}$,

$\mathcal{H} C_{n}\left(\mathcal{P}\left[\not p, p^{-1}\right]\right)=\mathcal{H} C_{n}(\mathcal{P}) \oplus \mathcal{H} C_{n-1}(\mathcal{P}) \oplus \mathcal{H} \mathcal{H}_{n}^{\infty}(\mathcal{P})$

The polynomial can be expressed using the matrix if all its coefficients are matrix and called polynomial matrices [19]. It can be mathematically expressed as follows:

$$
\begin{gathered}
p=\sum_{n=0}^{z} A(n) x^{n} \\
=A(0)+A(1) x+A(2) x^{2}+\cdots+A(z) x^{z},
\end{gathered}
$$

where $(n)$ denotes a matrix of constant coefficients. Let $\mathcal{P}$ be an associative unital polynomial algebra over $\mathcal{K}$, and let $M_{m}(\mathcal{P})$ be matrices algebra with coefficients in $\mathcal{P}$ of order $m . M_{m}(\mathcal{P})$ undergoes an involution:

$$
y \mapsto y^{*}, y=\left(y_{i j}\right), \quad y^{*}=\left(y_{j i}^{*}\right) \in M_{m}(\mathcal{A}),
$$

where the $\mathcal{K}$-module homomorphism 


$$
T r_{n}: M_{m}(\mathcal{P})^{\otimes(n-1)} \rightarrow \mathcal{P}^{\otimes(n-1)} .
$$

\section{Theorem 2-1:}

Let $\mathcal{P}^{\prime} \subseteq \mathcal{P}$ is a polynomial algebra. The long exact sequence is then obtained as follows:

$$
\begin{gathered}
\cdots \rightarrow \mathcal{H} \mathcal{H}_{n}\left(\mathcal{P}^{\prime}\right) \rightarrow \mathcal{H} \mathcal{H}_{n}(\mathcal{P}) \rightarrow \mathcal{H} \mathcal{H}_{n}\left(\mathcal{P} / \mathcal{P}^{\prime}\right) \rightarrow \\
\mathcal{H H}_{n-1}\left(\mathcal{P}^{\prime}\right) \rightarrow \cdots .
\end{gathered}
$$

Proof: See [14].

\section{Theory 2-2:}

If $\mathcal{P}^{\prime} \subseteq \mathcal{P}, \quad$ long exact sequence for cyclic (co)homology of polynomial algebra is

$$
\begin{gathered}
\cdots \rightarrow \mathcal{H} C_{n}\left(\mathcal{P}^{\prime}\right) \rightarrow \mathcal{H} C_{n}(\mathcal{P}) \rightarrow \mathcal{H} C_{n}\left(\mathcal{P} / \mathcal{P}^{\prime}\right) \rightarrow \\
\mathcal{H} C_{n-1}\left(\mathcal{P}^{\prime}\right) \rightarrow \cdots .
\end{gathered}
$$

Proof: See [20].

\section{Theory 2-3.}

The long exact sequences are known as Connes' exact periodicity sequences;

$$
\begin{gathered}
\quad \cdots \rightarrow \mathcal{H} \mathrm{C}_{n-1}(\mathcal{P}) \stackrel{\mathcal{B}}{\rightarrow} \mathcal{H} \mathcal{H}_{n}(\mathcal{P}) \stackrel{I}{\rightarrow} \mathcal{H} \mathrm{C}_{n}(\mathcal{P}) \stackrel{s}{\rightarrow} \\
\mathcal{H} \mathrm{C}_{n-2}(\mathcal{P}) \stackrel{\mathcal{B}}{\rightarrow} \mathcal{H} \mathcal{H}_{n-1}(\mathcal{P}) \rightarrow \cdots,
\end{gathered}
$$

Where; $\mathcal{J}$ is an inclusion map, $\mathcal{B}$ is a boundary map and $S$ is the periodicity map.

Proof: See [14].

\section{Theory 2-4:}

If $I$ is $H$-unital $k$-algebra, then the algebra of matrices $M_{r}(I)$ is $H$-untial, where

$$
\text { tr: } \mathcal{H H}_{n}\left(M_{r}(I), M_{r}(\mathcal{P})\right) \rightarrow \mathcal{H H}_{n}(I, \mathcal{P})
$$

and inc: $\mathcal{H H}_{n}(I, \mathcal{P}) \rightarrow \mathcal{H}_{\mathcal{H}}\left(M_{r}(I), M_{r}(\mathcal{P})\right)$ are isomorphism and inverse to each other.

Proof: See [3].

\section{Theorem 2-5:}

For any $H$-untial $\mathcal{K}$-algebra $\mathcal{P}$, a map tr: $\mathcal{H} C_{n}\left(M_{r}(I), M_{r}(\mathcal{P})\right) \rightarrow \mathcal{H} C_{n}(I, \mathcal{P}) \quad$ is $\quad$ an isomorphism and is the inverse of

$$
\text { inc: } \mathcal{H} \mathrm{C}_{n}(I, \mathcal{P}) \rightarrow \mathcal{H} \mathrm{C}_{n}\left(M_{r}(I), M_{r}(\mathcal{P})\right) \text {. }
$$

Proof: See [3].

The following section will examine the dihedral homology of polynomial algebra. The relation between dihedral and cyclic homology will be introduced for polynomial algebra and Laurent polynomials algebra. Morita's property of dihedral homology and Morita's invariance for dihedral homology in polynomial algebra and Laurent polynomials algebra will be examined, which states the trace map is inverse to the inclusion map. Finally, the long sequence of the dihedral homology will be studied.

\section{Main Results}

Let $\mathcal{P}=\mathcal{K}[\mathcal{P}]$ be the dihedral submodule of the dihedral module $\mathcal{P}_{\alpha}^{\mathcal{D}}$ generated by Polynomial $p_{1}, \cdots, p_{2} \in \mathcal{P}$, with involution $\left(\sum_{i} b_{i} y^{i}\right)^{*}=\sum_{i} b_{i}^{*} y^{i}, b_{i} \in \mathcal{P}$. The dihedral homology and cohomology of the group can be defined as

$$
\begin{gathered}
{ }^{\alpha} \mathcal{H} \mathcal{D}_{n}(\mathcal{P})=\operatorname{Tor}_{n}^{k[E]^{o p}}\left(k^{\mathcal{D}}, E \mathcal{P}_{\alpha}^{\mathcal{D}}\right), \\
{ }_{\alpha} \mathcal{H} \mathcal{D}^{n}(\mathcal{P})=\operatorname{Ext}_{k[E]}^{n}\left(E \mathcal{P}_{\alpha}^{\mathcal{D}}, k^{\mathcal{D}}\right) .
\end{gathered}
$$

If $\mathcal{P}$ is an involutive Polynomial algebra, then $\mathcal{H} D_{n}(\mathcal{P})=\mathcal{H}_{n}\left(\operatorname{Tot} \mathrm{CC}^{+}(\mathcal{P})\right)$ is the dihedral homology of $\mathcal{P}$, and the skew-dihedral homology of $\mathcal{P}$ is

$$
\mathcal{H} D_{n}^{\prime}(\mathcal{P})=\mathcal{H}_{n}\left(\operatorname{TotCC}^{-}(\mathcal{P})\right) \text {. }
$$

There is a canonical splitting of cyclic homology, which follows directly from the preceding definition:

$$
\mathcal{H} C_{n}(\mathcal{P})=\mathcal{H} \mathcal{D}_{n}(\mathcal{P}) \oplus \mathcal{H} \mathcal{D}_{n}^{\prime}(\mathcal{P}) \forall n .
$$

The direct sum of the following two exact sequences breaks up Connes' periodicity exact sequence naturally:

$$
\begin{aligned}
& \cdots \rightarrow \mathcal{H H}_{n}^{-} \rightarrow \mathcal{H D}_{n} \rightarrow \mathcal{H D}_{n-2}^{\prime} \rightarrow \mathcal{H H}_{n-1}^{+} \rightarrow \mathcal{H D}_{n-1}^{\prime} \rightarrow \\
& \mathcal{H D}_{n-3} \rightarrow \mathcal{H}^{\prime} \mathcal{H}_{n-2}^{-} \rightarrow \cdots, \\
& \cdots \rightarrow \mathcal{H H}_{n}^{+} \rightarrow \mathcal{H} \mathcal{D}_{n}^{\prime} \rightarrow \mathcal{H} \mathcal{D}_{n-2} \rightarrow \mathcal{H H}_{n-1}^{-} \rightarrow \mathcal{H} \mathcal{D}_{n-1} \rightarrow \\
& \mathcal{H D}_{n-3}^{\prime} \rightarrow \mathcal{H}^{\mathcal{H}_{n-2}^{+}} \rightarrow \cdots \text {, }
\end{aligned}
$$

when $\mathcal{K}$ contains $\mathbb{Q}$ :

$$
\begin{gathered}
\mathcal{H} \mathcal{D}_{n}=\mathcal{H} \mathrm{C}_{n}^{(1)} \oplus \mathcal{H} \mathrm{C}_{n}^{(3)} \oplus \cdots, \\
\text { and } \mathcal{H} \mathcal{D}_{n}^{\prime}=\mathcal{H} \mathrm{C}_{n}^{(0)} \oplus \mathcal{H} \mathrm{C}_{n}^{(2)} \oplus \cdots .
\end{gathered}
$$

If $\mathcal{P}$ is Laurent polynomial algebra $\mathcal{K}\left[p, p^{-1}\right]$, with the natural involution:

$$
\begin{aligned}
& \left(\sum_{i} a_{i} y^{i}+\sum_{j} b_{j} y^{-j}\right)^{*}=\sum_{i} a_{i}^{*} y^{i}+\sum_{j} b_{j}^{*} y^{-j} \forall a_{i}, b_{j} \in \\
& \mathcal{P} \text {. }
\end{aligned}
$$

Then we can define the dihedral of Laurent polynomial algebra by a formula;

$$
\begin{gathered}
{ }^{\alpha} \mathcal{H} \mathcal{D}_{n}\left(\mathcal{P}\left[p, p^{-1}\right]\right)= \\
{ }^{\alpha} \mathcal{H} \mathcal{D}_{n}(\mathcal{P}) \otimes^{\alpha} \mathcal{H} \mathcal{D}_{n-1}(\mathcal{P}) \otimes \mathcal{H} \mathcal{R}_{n}^{\infty}(\mathcal{P}) .
\end{gathered}
$$

If $\mathcal{K}$ contains $\mathbb{Q}$, the last relation given as;

$$
\mathcal{H C}_{n}(\mathcal{P}) \cong{ }^{\alpha} \mathcal{H} \mathcal{D}_{n}(\mathcal{P}) \oplus^{-\alpha} \mathcal{H} \mathcal{D}_{n}(\mathcal{P}), \alpha= \pm 1
$$

will be shown in the following theory.

\section{Theorem 3-1:}

If $\mathcal{P}$ is a polynomial algebra, then there is an isomorphism dihedral and cyclic homology;

$$
\mathcal{H} C_{n}(\mathcal{P}) \cong{ }^{\alpha} \mathcal{H} \mathcal{D}_{n}(\mathcal{P}) \oplus^{-\alpha} \mathcal{H} \mathcal{D}_{n}(\mathcal{P}), \alpha= \pm 1 .
$$

\section{Proof:}

If $\mathcal{K}$ is a commutative ring with unital, then the long 
exact sequence for dihedral and cyclic homology groups is

$$
\begin{gathered}
\cdots \rightarrow-\alpha \mathcal{H} \mathcal{D}_{n}(\mathcal{P}) \stackrel{g_{*}}{\rightarrow} \mathcal{H} C_{n}(\mathcal{P}) \stackrel{i_{*}}{\rightarrow} \alpha \mathcal{H} \mathcal{D}_{n}(\mathcal{P}) \rightarrow \\
-\alpha \mathcal{H} \mathcal{D}_{n-1}(\mathcal{P}) \rightarrow \cdots,
\end{gathered}
$$

where $g_{*}$ is homomorphism. When $\frac{1}{2} \in \mathcal{K}$ is used, the above exact sequence is broken down into short exact sequences:

$$
0 \rightarrow^{-\alpha} \mathcal{H} \mathcal{D}_{n}(\mathcal{P}) \stackrel{g_{*}}{\rightarrow} \mathcal{H} C_{n}(\mathcal{P}) \stackrel{i_{*}}{\rightarrow} \alpha \mathcal{H} \mathcal{D}_{n}(\mathcal{P}) \rightarrow 0
$$

We can also express the sequence by an infinite commutative diagram of exact columns and rows:

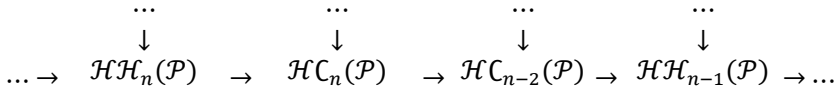

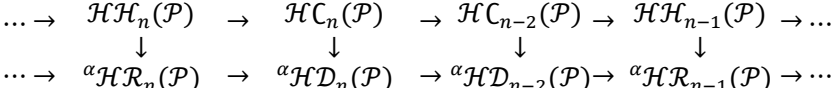

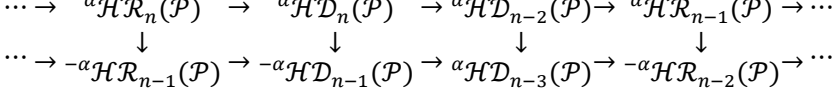

$$
\begin{aligned}
& \cdots \rightarrow \underset{\mathcal{H H}_{n-1}(\mathcal{P})}{\downarrow} \rightarrow \underset{\mathcal{H C}_{n-1}(\mathcal{P})}{\downarrow} \rightarrow \underset{\mathcal{H C}_{n-3}(\mathcal{P})}{\downarrow} \rightarrow \underset{\mathcal{H H}_{n-2}(\mathcal{P})}{\downarrow} \rightarrow \cdots \\
& \begin{array}{llll}
\downarrow & \downarrow & \downarrow & \downarrow \\
\ldots & \ldots & \ldots & \ldots
\end{array}
\end{aligned}
$$

we have

we have

$$
\mathcal{H}_{*}(B G+B \mathcal{O}(2) ; \mathcal{K}) \cong
$$

$$
\mathcal{H}_{*}(\mathcal{P} ; \mathcal{K}) \otimes \mathcal{H}_{*}(B \mathcal{O}(2) ; \mathcal{K}) \cong \mathcal{H}_{*}(\mathcal{P} ; \mathcal{K}) \otimes \mathcal{K}[t],
$$

implying the first isomorphism, when

$$
\begin{gathered}
\mathcal{H}_{*}(B \mathcal{O}(2)) \simeq \mathcal{K}[t] . \text { If } \frac{1}{2} \in \mathcal{K} \text { and } \alpha= \pm 1, \\
\mathcal{H} C_{n}(\mathcal{P}) \cong \mathcal{H}_{n}(\mathcal{P} ; \mathcal{K}) \oplus \mathcal{H}_{n-2}(\mathcal{P} ; \mathcal{K}) \oplus \mathcal{H}_{n-4}(\mathcal{P} ; \mathcal{K}) \\
\bigoplus \cdots,
\end{gathered}
$$

we have the following natural isomorphisms hold:

$$
\begin{gathered}
{ }^{1} \mathcal{H} \mathcal{D}_{n}(\mathcal{P}) \cong \\
\mathcal{H}_{n}(\mathcal{P} ; \mathcal{K}) \oplus \mathcal{H}_{n-4}(\mathcal{P} ; \mathcal{K}) \oplus \mathcal{H}_{n-8}(\mathcal{P} ; \mathcal{K}) \oplus \cdots \\
{ }^{-1} \mathcal{H} \mathcal{D}_{n}(\mathcal{P}) \cong \\
\mathcal{H}_{n-2}(\mathcal{P} ; \mathcal{K}) \oplus \mathcal{H}_{n-6}(\mathcal{P} ; \mathcal{K}) \oplus \mathcal{H}_{n-10}(\mathcal{P} ; \mathcal{K}) \oplus \cdots
\end{gathered}
$$

From equations (3) and (4), we get

$$
\mathcal{H} C_{n}(\mathcal{P}) \cong{ }^{1} \mathcal{H} \mathcal{D}_{n}(\mathcal{P}) \oplus^{-1} \mathcal{H} \mathcal{D}_{n}(\mathcal{P}),
$$

implying the second isomorphism.

In the following theory, the relation between dihedral and cyclic homology of Laurent polynomial algebras will be discussed.

\section{Theorem 3-2:}

If $\mathcal{P}$ is a Laurent polynomial algebra $\mathcal{K}\left[p, p^{-1}\right]$, then we have;

$$
\begin{aligned}
& \mathcal{H} C_{n}\left(\mathcal{P}\left[p, p^{-1}\right]\right) \cong \\
& \alpha \mathcal{H} \mathcal{D}_{n}\left(\mathcal{P}\left[p, p^{-1}\right]\right) \oplus^{-\alpha} \mathcal{H} \mathcal{D}_{n}\left(\mathcal{P}\left[p, p^{-1}\right]\right), \quad \alpha= \pm 1
\end{aligned}
$$

\section{Proof:}

From the definition and we take $\alpha= \pm 1$ then the following hold:

$$
\begin{gathered}
{ }^{1} \mathcal{H} \mathcal{D}_{n}\left(\mathcal{P}\left[p, p^{-1}\right]\right) \\
\cong \mathcal{H}_{n}(\mathcal{P} ; \mathcal{K}) \oplus \mathcal{H}_{n-4}(\mathcal{P} ; \mathcal{K}) \oplus \mathcal{H}_{n-8}(\mathcal{P} ; \mathcal{K}) \oplus \cdots, \\
{ }^{1} \mathcal{H}_{n}\left(\mathcal{P}\left[p, p^{-1}\right]\right)= \\
{ }^{1} \mathcal{H} \mathcal{D}_{n}(\mathcal{P}) \oplus{ }^{1} \mathcal{H} \mathcal{D}_{n-1}(\mathcal{P}) \otimes \mathcal{H} \mathcal{R}_{n}^{\infty}(\mathcal{P})
\end{gathered}
$$

From equations (5) and (6) we get

$$
\begin{gathered}
{ }^{1} \mathcal{H}_{n}(\mathcal{P}) \oplus{ }^{1} \mathcal{H} \mathcal{D}_{n-1}(\mathcal{P}) \otimes \mathcal{H} \mathcal{R}_{n}^{\infty}(\mathcal{P}) \\
\cong \mathcal{H}_{n}(\mathcal{P} ; \mathcal{K}) \oplus \mathcal{H}_{n-4}(\mathcal{P} ; \mathcal{K}) \oplus \mathcal{H}_{n-8}(\mathcal{P} ; \mathcal{K}) \oplus \cdots
\end{gathered}
$$

Similarly, from the following

$$
\begin{gathered}
{ }^{-1} \mathcal{H} \mathcal{D}_{n}\left(\mathcal{P}\left[p, p^{-1}\right]\right) \cong \\
\mathcal{H}_{n-2}(\mathcal{P} ; \mathcal{K}) \mathcal{H}_{n-6}(\mathcal{P} ; \mathcal{K}) \oplus \mathcal{H}_{n-10}(\mathcal{P} ; \mathcal{K}) \oplus \cdots \\
-1 \mathcal{H} \mathcal{D}_{n}\left(\mathcal{P}\left[p, p^{-1}\right]\right)= \\
{ }^{-1} \mathcal{H} \mathcal{D}_{n}(\mathcal{P}) \oplus^{-1} \mathcal{H} \mathcal{D}_{n-1}(\mathcal{P}) \otimes \mathcal{H} \mathcal{R}_{n}^{\infty}(\mathcal{P}),
\end{gathered}
$$

we get

$$
{ }^{-1} \mathcal{H} \mathcal{D}_{n}(\mathcal{P}) \bigoplus^{-1} \mathcal{H} \mathcal{D}_{n-1}(\mathcal{P}) \otimes \mathcal{H} \mathcal{R}_{n}^{\infty}(\mathcal{P}) \cong
$$$$
\mathcal{H}_{n-2}(\mathcal{P} ; \mathcal{K}) \oplus \mathcal{H}_{n-6}(\mathcal{P} ; \mathcal{K}) \oplus \mathcal{H}_{n-10}(\mathcal{P} ; \mathcal{K}) \oplus \cdots .
$$

We know that

$$
\begin{gathered}
\mathcal{H} C_{n}\left(\mathcal{P}\left[p, p^{-1}\right]\right) \cong \\
\mathcal{H}_{n}(\mathcal{P} ; \mathcal{K}) \oplus \mathcal{H}_{n-2}(\mathcal{P} ; \mathcal{K}) \oplus \mathcal{H}_{n-4}(\mathcal{P} ; \mathcal{K}) .
\end{gathered}
$$

From equations (7), (10), and (11), we get

$$
\begin{gathered}
\mathcal{H} C_{n}\left(\mathcal{P}\left[p, p^{-1}\right]\right) \cong \\
{ }^{1} \mathcal{H} \mathcal{D}_{n}\left(\mathcal{P}\left[p, p^{-1}\right]\right) \bigoplus^{-1} \mathcal{H} \mathcal{D}_{n}\left(\mathcal{P}\left[p, p^{-1}\right]\right) .
\end{gathered}
$$

In the following theorem, we prove the Morita Invariance of polynomial algebra.

\section{Theorem 3-3:}

If $\mathcal{P}$ is a polynomial algebra over $\mathcal{K}$ and $M_{m}(\mathcal{P})$ is the polynomial algebra of matrices, then the map

$$
\operatorname{Tr}_{*}:{ }^{\alpha} \mathcal{H} \mathcal{D}_{n}\left(M_{m}(\mathcal{P})\right) \rightarrow{ }^{\alpha} \mathcal{H} \mathcal{D}_{n}(\mathcal{P})
$$

is an isomorphism for all $m \geq 1$ and $n \geq 0$.

\section{Proof:}

Assume that the bicomplex ${ }^{\alpha} \Lambda(\mathcal{P})=\Lambda\left(\mathcal{P}_{\alpha}^{\mathcal{D}}\right)$ has the group $\mathbb{Z} / 2$.

If $j$ natural projection,

$$
\left(\operatorname{Tot}^{\alpha} \Lambda\left(M_{m}(\mathcal{P})\right)[-2]\right)_{n}=\left(\operatorname{Tot}^{\alpha} \Lambda\left(M_{m}(\mathcal{P})\right)\right)_{n-2},
$$

$$
i:{ }^{\alpha} \mathcal{R}\left(M_{m}(\mathcal{P})\right) \rightarrow \operatorname{Tot}^{\alpha} \Lambda\left(M_{m}(\mathcal{P})\right)
$$

and ${ }^{\alpha} \mathcal{R}\left(M_{m}(\mathcal{P})\right)=\operatorname{ker} j$, then the following short sequence of $\mathbb{Z} / 2$-complexes is exact:

$$
\begin{aligned}
0 \rightarrow{ }^{\alpha} & \mathcal{R}\left(M_{m}(\mathcal{P})\right) \stackrel{i}{\rightarrow} \operatorname{Tot}^{\alpha} \Lambda\left(M_{m}(\mathcal{P})\right) \\
& \stackrel{j}{\rightarrow} \operatorname{Tot}^{\alpha} \Lambda\left(M_{m}(\mathcal{P})\right)[-2] \rightarrow 0 .
\end{aligned}
$$

This short sequence's hyperhomology yields the long exact sequence; 


$$
\begin{gathered}
\rightarrow \mathcal{H}_{n}\left(\mathbb{Z} / 2,{ }^{\alpha} \mathcal{R}\left(M_{m}(\mathcal{P})\right)\right) \stackrel{i}{\rightarrow} \mathcal{H}_{n}\left(\mathbb{Z} / 2, \operatorname{Tot}^{\alpha} \Lambda\left(M_{m}(\mathcal{P})\right)\right) \stackrel{j}{\rightarrow} \\
\mathcal{H}_{n}\left(\mathbb{Z} . / 2, \operatorname{Tot}^{\alpha} \Lambda\left(M_{m}(\mathcal{P})\right)[-2]\right) \rightarrow \\
\mathcal{H}_{n}\left(\mathbb{Z} / 2,{ }^{\alpha} \mathcal{R}\left(M_{m}(\mathcal{P})\right)\right) \rightarrow \cdots .
\end{gathered}
$$

If $\operatorname{Tr}$ is a homomorphism between $\mathcal{H}_{n}\left(\operatorname{Tot}^{\alpha} \Lambda\left(M_{m}(\mathcal{P})\right)\right)$ and $\mathcal{H}_{n}\left(\operatorname{Tot}^{\alpha} \Lambda(\mathcal{P})\right)$, then

$$
\begin{gathered}
\mathcal{H}_{n}\left(\operatorname{Tot}^{\alpha} \Lambda\left(M_{m}(\mathcal{P})\right)\right)=\mathcal{H} C_{n}\left(M_{m}(\mathcal{P})\right), \\
\mathcal{H}_{n}\left(\operatorname{Tot}^{\alpha} \Lambda(\mathcal{P})\right)=\mathcal{H} C_{n}(\mathcal{P}) .
\end{gathered}
$$

Applying $\operatorname{Tr}: M_{m}(\mathcal{P}) \rightarrow \mathcal{P}$ on the cyclic homology, we get

$$
\operatorname{Tr}: \mathcal{H} C_{n}\left(M_{m}(\mathcal{P})\right) \rightarrow \mathcal{H} C_{n}(\mathcal{P}) \text {. }
$$

If we apply $\operatorname{Tr}: M_{m}(\mathcal{P}) \rightarrow \mathcal{P}$ on the dihedral homology with consideration;

$$
\begin{gathered}
{ }^{\alpha} \mathcal{H D}_{n}\left(M_{m}(\mathcal{P})\right)=\mathcal{H}_{n}\left(\mathbb{Z} / 2,{ }^{\alpha} \Lambda\left(M_{m}(\mathcal{P})\right)\right), \\
{ }^{\alpha} \mathcal{H D}_{n}(\mathcal{P})=\mathcal{H}_{n}\left(\mathbb{Z} / 2,{ }^{\alpha} \Lambda(\mathcal{P})\right),
\end{gathered}
$$

we get

$$
\operatorname{Tr}_{*}:{ }^{\alpha} \mathcal{H} \mathcal{D}_{n}\left(M_{m}(\mathcal{P})\right) \rightarrow{ }^{\alpha} \mathcal{H} \mathcal{D}_{n}(\mathcal{P})
$$

In the following theorem, we prove the inclusion map of the Laurent polynomial algebra.

\section{Theorem 3-4:}

If $\mathcal{P}$ is Laurent polynomial algebra over $\mathcal{K}\left[\not p, p^{-1}\right]$ and $M_{m}\left(\mathcal{P}\left[\not, p^{-1}\right]\right)$ is the Laurent polynomial algebra of matrices, then the map

$$
\text { inc: }{ }^{\alpha} \mathcal{H} \mathcal{D}_{n}\left(\mathcal{P}\left[\not p, p^{-1}\right]\right) \rightarrow{ }^{\alpha} \mathcal{H} \mathcal{D}_{n}\left(M_{m}\left(\mathcal{P}\left[\not p, p^{-1}\right]\right)\right)
$$

is an isomorphism for all $m \geq 1$ and $n \geq 0$.

\section{Proof:}

Suppose that bicomplex $\Lambda\left(\mathcal{P}_{\alpha}^{\mathcal{D}}\right)={ }^{\alpha} \Lambda(\mathcal{P})$ has a fixed action of the group $\mathbb{Z} / 2$.

$$
\begin{aligned}
& \text { If } i:{ }^{\alpha} \mathcal{R}\left(M_{m}\left(\mathcal{P}\left[\not p, p^{-1}\right]\right)\right) \rightarrow \operatorname{Tot}^{\alpha} \Lambda\left(M_{m}\left(\mathcal{P}\left[p, p^{-1}\right]\right)\right) \text {, } \\
& j: \operatorname{Tot}^{\alpha} \Lambda\left(M_{m}\left(\mathcal{P}\left[p, p^{-1}\right]\right)\right) \rightarrow \\
& \operatorname{Tot}^{\alpha} \Lambda\left(M_{m}\left(\mathcal{P}\left[p, p^{-1}\right]\right)\right)[-2],
\end{aligned}
$$

and ${ }^{\alpha} \mathcal{R}\left(M_{m}\left(\mathcal{P}\left[p, p^{-1}\right]\right)\right)=\operatorname{ker} j$, where $j$ natural projection, then the short sequence of $\mathbb{Z} / 2$-complexes after that is

$$
\begin{aligned}
0 \rightarrow{ }^{\alpha} & \mathcal{R}\left(M_{m}\left(\mathcal{P}\left[p, p^{-1}\right]\right)\right) \stackrel{i}{\rightarrow} \operatorname{Tot}^{\alpha} \Lambda\left(M_{m}\left(\mathcal{P}\left[p, p^{-1}\right]\right)\right) \\
\stackrel{j}{\rightarrow} & T o t^{\alpha} \Lambda\left(M_{m}\left(\mathcal{P}\left[p, p^{-1}\right]\right)\right)[-2] \rightarrow 0 .
\end{aligned}
$$

The hyper-homology of the upper short exact sequence gives a long sequence;

$$
\cdots \rightarrow \mathcal{H}_{n}\left(\mathbb{Z} / 2,{ }^{\alpha} \mathcal{R}\left(M_{m}\left(\mathcal{P}\left[p, p^{-1}\right]\right)\right)\right) \stackrel{i}{\rightarrow}
$$

$$
\begin{gathered}
\mathcal{H}_{n}\left(\mathbb{Z} / 2, \operatorname{Tot}^{\alpha} \Lambda\left(M_{m}\left(\mathcal{P}\left[\not p, p^{-1}\right]\right)\right)\right) \\
\stackrel{j}{\rightarrow} \mathcal{H}_{n}\left(\mathbb{Z} / 2, \operatorname{Tot}^{\alpha} \Lambda\left(M_{m}\left(\mathcal{P}\left[\not p, p^{-1}\right]\right)\right)[-2]\right) \rightarrow \\
\quad \mathcal{H}_{n}\left(\mathbb{Z} / 2,{ }^{\alpha} \mathcal{R}\left(M_{m}\left(\mathcal{P}\left[\not p, p^{-1}\right]\right)\right)\right) \rightarrow \cdots .
\end{gathered}
$$

If $i n c$ is a homomorphism between

$$
\mathcal{H}_{n}\left(\operatorname{Tot}^{\alpha} \Lambda\left(\mathcal{P}\left[p, p^{-1}\right]\right)\right)
$$

and

$$
\mathcal{H}_{n}\left(\operatorname{Tot}^{\alpha} \Lambda\left(M_{m}\left(\mathcal{P}\left[\not p, p^{-1}\right]\right)\right)\right),
$$

then

$$
\begin{gathered}
\mathcal{H}_{n}\left(\operatorname{Tot}^{\alpha} \Lambda\left(M_{m}\left(\mathcal{P}\left[p, p^{-1}\right]\right)\right)\right) \\
=\mathcal{H} C_{n}\left(M_{m}\left(\mathcal{P}\left[\not p, p^{-1}\right]\right)\right), \\
\mathcal{H}_{n}\left(\operatorname{Tot}^{\alpha} \Lambda\left(\mathcal{P}\left[\not p, p^{-1}\right]\right)\right)=\mathcal{H} C_{n}\left(\mathcal{P}\left[\not p, p^{-1}\right]\right) .
\end{gathered}
$$

If we take the cyclic of;

$$
\text { inc: } P\left[\not p, p^{-1}\right] \rightarrow M_{m}\left(\mathcal{P}\left[\not p, p^{-1}\right]\right),
$$

we obtain

$$
\text { inc: } \mathcal{H} C_{n}\left(\mathcal{P}\left[\not p, p^{-1}\right]\right) \rightarrow \mathcal{H C} C_{n}\left(M_{m}\left(\mathcal{P}\left[\not p, p^{-1}\right]\right)\right) \text {. }
$$

If we apply the upper case in a dihedral homology, considering

$$
\begin{gathered}
{ }^{\alpha} \mathcal{H}_{n}\left(M_{m}\left(\mathcal{P}\left[p, p^{-1}\right]\right)\right)= \\
\mathcal{H}_{n}\left(\mathbb{Z} / 2,{ }^{\alpha} \Lambda\left(M_{m}\left(\mathcal{P}\left[p, p^{-1}\right]\right)\right)\right), \\
{ }^{\alpha} \mathcal{H} \mathcal{D}_{n}\left(\left(\mathcal{P}\left[p, p^{-1}\right]\right)\right)=\mathcal{H}_{n}\left(\mathbb{Z} / 2,{ }^{\alpha} \Lambda\left(\left(\mathcal{P}\left[p, p^{-1}\right]\right)\right)\right),
\end{gathered}
$$

we get

inc: ${ }^{\alpha} \mathcal{H} \mathcal{D}_{n}\left(\left(\mathcal{P}\left[p, p^{-1}\right]\right)\right) \rightarrow{ }^{\alpha} \mathcal{H} \mathcal{D}_{n}\left(M_{m}\left(\left(\mathcal{P}\left[p, p^{-1}\right]\right)\right)\right)$.

In the following theory, we will demonstrate that the trace map of dihedral is inverse of the inclusion map.

\section{Theorem 3-5:}

Consider that $\mathcal{P}$ is polynomial algebra and $I$ is $H$-untial over $\mathcal{K}$. Assume

$$
\text { tr: }{ }^{\alpha} \mathcal{H} \mathcal{D}_{n}(\mathcal{P} \otimes I) \rightarrow{ }^{\alpha} \mathcal{H} \mathcal{D}_{n}\left(M_{m}(\mathcal{P}) \otimes M_{m}(I)\right)
$$

and

$$
\text { inc: }{ }^{\alpha} \mathcal{H} \mathcal{D}_{n}\left(M_{m}(\mathcal{P}) \otimes M_{m}(I) \rightarrow{ }^{\alpha} \mathcal{H} \mathcal{D}_{n}(\mathcal{P} \otimes I)\right.
$$

are used to define these maps $a \rightarrow\left(\begin{array}{cccc}a & & & \\ & 0 & & \\ & & \ddots & \\ & & & 0\end{array}\right)$ and $\operatorname{inc}\left(\left(a_{i j}\right) \otimes\left(b_{i j}\right)\right)=\sum_{i, j} a_{i j} \otimes b_{j i}$, respectively.

Accordingly, the map

$\alpha_{\mathcal{H}} \mathcal{D}_{n}\left(M_{m}(\mathcal{P}) \otimes M_{m}(I)\right) \stackrel{\text { inc }}{\rightarrow} \alpha_{\mathcal{H}} \mathcal{D}_{n}(\mathcal{P} \otimes I) \quad$ inverses the map

$$
\alpha \mathcal{H} \mathcal{D}_{n}(\mathcal{P} \otimes I) \stackrel{\text { tr }}{\rightarrow} \alpha \mathcal{H} \mathcal{D}_{n}\left(M_{m}(\mathcal{P}) \otimes M_{m}(I)\right) .
$$




\section{Proof:}

The following commutative diagram is the extension morphism;

$$
\begin{array}{ccccccccc}
0 & \rightarrow & I & \rightarrow & I_{+} & \rightarrow & \mathcal{K} & \rightarrow & 0 \\
& & \downarrow & & \downarrow & & \downarrow & & \\
0 & \rightarrow & M_{m}(I) & \rightarrow & M_{m}\left(I_{+}\right) & \rightarrow & M_{m}(\mathcal{K}) & \rightarrow & 0
\end{array},
$$

where the vertical and horizontal maps are executed. Isomorphism can be seen in the left vertical arrow by extension property of polynomial algebra, and the rows are accurate. If we consider the upper diagram's dihedral homology, then we obtain

$$
\begin{aligned}
& 0 \rightarrow \alpha \mathcal{H} \mathcal{D}_{n}(I) \quad \rightarrow \quad \alpha \mathcal{H} \mathcal{D}_{n}\left(I_{+}\right) \quad \rightarrow \quad \alpha \mathcal{H} \mathcal{D}_{n}(\mathcal{K}) \quad \rightarrow 0 \\
& \downarrow \quad \downarrow \quad \downarrow \\
& 0 \rightarrow{ }^{\alpha} \mathcal{H} \mathcal{D}_{n}\left(M_{m}(I)\right) \rightarrow^{\alpha} \mathcal{H} \mathcal{D}_{n}\left(M_{m}\left(I_{+}\right)\right) \rightarrow^{\alpha} \mathcal{H} \mathcal{D}_{n}\left(M_{m}(\mathcal{K})\right) \rightarrow 0
\end{aligned}
$$

Consequently, the right vertical arrow is isomorphic, and we get a commutative diagram;

$$
\begin{array}{cccc}
\alpha_{\mathcal{H}} \mathcal{D}_{n}\left(M_{m}(\mathcal{P}) \otimes M_{m}(I)\right) & \stackrel{\cong}{\rightarrow} \mathcal{H} \mathcal{D}_{n}\left(M_{m}(\mathcal{P}) \otimes M_{m}\left(I_{+}\right)\right) \\
\operatorname{tr} \downarrow & & \operatorname{tr} \downarrow \\
\alpha_{\mathcal{H} \mathcal{D}_{n}(\mathcal{P} \otimes I)} & \cong & \alpha_{\mathcal{H} \mathcal{D}_{n}\left(\mathcal{P} \otimes I_{+}\right)}^{\rightarrow} &
\end{array}
$$

If the right $t r$ is an isomorphism according to Morita invariance for unital polynomial algebra, then

$$
\text { tr }:{ }^{\alpha} \mathcal{H} \mathcal{D}_{n}\left(M_{m}(\mathcal{P}) \otimes M_{m}(I)\right) \rightarrow{ }^{\alpha} \mathcal{H} \mathcal{D}_{n}(\mathcal{P} \otimes I)
$$

is an isomorphism. Thus, inc is a right inverse of $t r$.

The theorem for dihedral (co)homology is introduced and proved in the next theory.

\section{Theorem 3-6:}

In Dihedral Homology, if we consider the exact short sequence $0 \rightarrow \mathcal{P} \rightarrow \mathcal{P}^{\prime} \rightarrow \mathcal{P}^{\prime \prime} \rightarrow 0$ of polynomials algebras over a field, we obtain the following long exact sequence;

$$
\begin{gathered}
\cdots \rightarrow \mathcal{H} \mathcal{D}_{n}(\mathcal{P}) \rightarrow \mathcal{H} \mathcal{D}_{n}\left(\mathcal{P}^{\prime}\right) \rightarrow \mathcal{H} \mathcal{D}_{n}\left(\mathcal{P}^{\prime \prime}\right) \rightarrow \\
\mathcal{H} \mathcal{D}_{n-1}(\mathcal{P}) \rightarrow \mathcal{H} \mathcal{D}_{n-1}\left(\mathcal{P}^{\prime}\right) \rightarrow \mathcal{H} \mathcal{D}_{n-1}\left(\mathcal{P}^{\prime \prime}\right) \rightarrow \cdots
\end{gathered}
$$

where $\mathcal{P}^{\prime \prime} \subset \mathcal{P}^{\prime} \subset \mathcal{P}$.

\section{Proof:}

For the algebra $\mathcal{P}^{\prime}$, we define the next short exact sequence;

$$
0 \rightarrow \mathcal{P} \mathcal{P}^{\prime} \rightarrow \mathcal{T} \mathcal{P}^{\prime} \rightarrow \mathcal{P}^{\prime} \rightarrow 0,
$$

where $\mathcal{T} \mathcal{P}^{\prime}$ is the algebra of non-unital involution polynomials over $\mathcal{P}$ and $\mathcal{P} \mathcal{P}^{\prime}$ is ideal in a unital polynomials algebra $\mathcal{T} \mathcal{P}^{\prime}$, then a long exact sequence is

$$
\begin{array}{r}
\cdots \rightarrow \mathcal{H} \mathcal{D}_{n}\left(\mathcal{P} \mathcal{P}^{\prime}\right) \rightarrow \mathcal{H} \mathcal{D}_{n}\left(\mathcal{T} \mathcal{P}^{\prime}\right) \rightarrow \mathcal{H} \mathcal{D}_{n}\left(\mathcal{P}^{\prime}\right) \rightarrow \\
\mathcal{H} \mathcal{D}_{n-1}\left(\mathcal{P} \mathcal{P}^{\prime}\right) \rightarrow \mathcal{H} \mathcal{D}_{n-1}\left(\mathcal{T} \mathcal{P}^{\prime}\right) \rightarrow \mathcal{H} \mathcal{D}_{n-1}\left(\mathcal{P}^{\prime}\right) \rightarrow \cdots .
\end{array}
$$

If $\mathcal{K}$ be a kernel of the following sequence

$$
\begin{gathered}
0 \rightarrow \mathcal{K} \rightarrow \mathcal{T} \mathcal{P}^{\prime} \rightarrow \mathcal{P}^{\prime \prime} \rightarrow 0 \\
\cdots \rightarrow \mathcal{H} \mathcal{D}_{n}(\mathcal{K}) \rightarrow \mathcal{H} \mathcal{D}_{n}\left(\mathcal{T} \mathcal{P}^{\prime}\right) \rightarrow \mathcal{H} \mathcal{D}_{n}\left(\mathcal{P}^{\prime \prime}\right) \rightarrow \\
\mathcal{H} \mathcal{D}_{n-1}(\mathcal{K}) \rightarrow \mathcal{H} \mathcal{D}_{n-1}\left(\mathcal{T} \mathcal{P}^{\prime}\right) \rightarrow \mathcal{H} \mathcal{D}_{n-1}\left(\mathcal{P}^{\prime \prime}\right) \rightarrow \cdots, \quad(13)
\end{gathered}
$$

where $\mathcal{K}$ is the free algebra of unital polynomials $\mathcal{T} \mathcal{P}^{\prime}$, then $\mathcal{P} \mathcal{P}^{\prime}$ and $\mathcal{K}$ are roughly $H$-unital from a long exact sequence for the sequences $(12,13)$.

We have

$$
\mathcal{H} \mathcal{D}_{n-1}\left(\mathcal{P} \mathcal{P}^{\prime}\right) \cong \mathcal{H} \mathcal{D}_{n}\left(\mathcal{P}^{\prime}\right) \& \mathcal{H} \mathcal{D}_{n-1}(\mathcal{K}) \cong \mathcal{H} \mathcal{D}_{n}\left(\mathcal{P}^{\prime \prime}\right)
$$

Suppose that the next short sequence is

$$
\begin{gathered}
0 \rightarrow \mathcal{P} \mathcal{P}^{\prime} \rightarrow \mathcal{K} \rightarrow \mathcal{P} \rightarrow 0 \\
\cdots \rightarrow \mathcal{H} \mathcal{D}_{n}\left(\mathcal{P} \mathcal{P}^{\prime}\right) \rightarrow \mathcal{H} \mathcal{D}_{n}(\mathcal{K}) \rightarrow \mathcal{H} \mathcal{D}_{n}(\mathcal{P}) \rightarrow \mathcal{H} \mathcal{D}_{n-1}\left(\mathcal{P} \mathcal{P}^{\prime}\right) \rightarrow \\
\mathcal{H D}_{n-1}(\mathcal{K}) \rightarrow \mathcal{H} \mathcal{D}_{n-1}(\mathcal{P}) \rightarrow \cdots .
\end{gathered}
$$

The proof of our theorem comes from the long exact sequence.

The theorem for reflexive (co)homology is introduced and demonstrated in the next theory.

\section{Theorem 3-7:}

If there is an exact short sequence $0 \rightarrow \mathcal{P} \rightarrow \mathcal{P}^{\prime} \rightarrow$ $\mathcal{P}^{\prime \prime} \rightarrow 0$ of polynomials algebras over the field, then we have the next long exact sequence in hyperhomology

$$
\begin{gathered}
\cdots \rightarrow \mathcal{H} \mathcal{R}_{n}(\mathcal{P}) \rightarrow \mathcal{H} \mathcal{R}_{n}\left(\mathcal{P}^{\prime}\right) \rightarrow \mathcal{H} \mathcal{R}_{n}\left(\mathcal{P}^{\prime \prime}\right) \rightarrow \\
\mathcal{H} \mathcal{R}_{n-1}(\mathcal{P}) \rightarrow \mathcal{H} \mathcal{R}_{n-1}\left(\mathcal{P}^{\prime}\right) \rightarrow \mathcal{H} \mathcal{R}_{n-1}\left(\mathcal{P}^{\prime \prime}\right) \rightarrow \cdots
\end{gathered}
$$

where $\mathcal{P}^{\prime \prime} \subset \mathcal{P}^{\prime} \subset \mathcal{P}$.

\section{Proof:}

Let $\mathcal{T} \mathcal{P}^{\prime}$ be the algebra of non-unital involution polynomials over $\mathcal{P}, \mathcal{P P}^{\prime}$ be the ideal in the unital polynomials algebra $\mathcal{T} \mathcal{P}^{\prime}$, and $\mathcal{K}$ be the kernel.

For the short exact sequence $0 \rightarrow \mathcal{P} \mathcal{P}^{\prime} \rightarrow \mathcal{T} \mathcal{P}^{\prime} \rightarrow \mathcal{P}^{\prime} \rightarrow$ 0 , we obtain long exact sequence as follows:

$$
\begin{aligned}
\cdots & \rightarrow \mathcal{H} \mathcal{R}_{n}\left(\mathcal{P} \mathcal{P}^{\prime}\right) \rightarrow \mathcal{H} \mathcal{R}_{n}\left(\mathcal{T P}^{\prime}\right) \rightarrow \mathcal{H} \mathcal{R}_{n}\left(\mathcal{P}^{\prime}\right) \rightarrow \\
\mathcal{H} \mathcal{R}_{n-1}\left(\mathcal{P} \mathcal{P}^{\prime}\right) & \rightarrow \mathcal{H} \mathcal{R}_{n-1}\left(\mathcal{T}^{\prime}\right) \rightarrow \mathcal{H} \mathcal{R}_{n-1}\left(\mathcal{P}^{\prime}\right) \rightarrow \cdots
\end{aligned}
$$

We get the long exact sequence for $0 \rightarrow \mathcal{K} \rightarrow \mathcal{T} \mathcal{P}^{\prime} \rightarrow$ $\mathcal{P}^{\prime \prime} \rightarrow 0$ as

$$
\begin{aligned}
\cdots & \rightarrow \mathcal{H} \mathcal{R}_{n}(\mathcal{K}) \rightarrow \mathcal{H} \mathcal{R}_{n}\left(\mathcal{T} \mathcal{P}^{\prime}\right) \rightarrow \mathcal{H} \mathcal{R}_{n}\left(\mathcal{P}^{\prime \prime}\right) \rightarrow \\
\mathcal{H} \mathcal{R}_{n-1}(\mathcal{K}) & \rightarrow \mathcal{H} \mathcal{R}_{n-1}\left(\mathcal{T} \mathcal{P}^{\prime}\right) \rightarrow \mathcal{H} \mathcal{R}_{n-1}\left(\mathcal{P}^{\prime \prime}\right) \rightarrow \cdots
\end{aligned}
$$

From equations (15) and (16), we get

$$
\begin{gathered}
\mathcal{H} \mathcal{R}_{n}\left(\mathcal{P}^{\prime}\right) \cong \mathcal{H} \mathcal{R}_{n-1}\left(\mathcal{P} \mathcal{P}^{\prime}\right) \\
\text { and } \mathcal{H} \mathcal{R}_{n}\left(\mathcal{P}^{\prime \prime}\right) \cong \mathcal{H} \mathcal{R}_{n-1}(\mathcal{K}) \text {. }
\end{gathered}
$$

If we take the short sequence $0 \rightarrow \mathcal{P} \mathcal{P}^{\prime} \rightarrow \mathcal{K} \rightarrow \mathcal{P} \rightarrow 0$, then the long exact sequence is as follows:

$$
\cdots \rightarrow \mathcal{H} \mathcal{R}_{n}\left(\mathcal{P} \mathcal{P}^{\prime}\right) \rightarrow \mathcal{H} \mathcal{R}_{n}(\mathcal{K}) \rightarrow \mathcal{H} \mathcal{R}_{n}(\mathcal{P}) \rightarrow
$$

$\mathcal{H} \mathcal{R}_{n-1}\left(\mathcal{P} \mathcal{P}^{\prime}\right) \rightarrow \mathcal{H} \mathcal{R}_{n-1}(\mathcal{K}) \rightarrow \mathcal{H} \mathcal{R}_{n-1}(\mathcal{P}) \rightarrow \cdots$

By applying the isomorphism in equation (17) to equation (18), we get the proof of our theory. 


\section{Conclusions}

We demonstrate the dihedral and reflexive homology of polynomial algebra. The relationship between dihedral and cyclic (co)homology for polynomial algebra was introduced, which is $\mathcal{H} C_{n}(\mathcal{P}) \cong{ }^{\alpha} \mathcal{H} \mathcal{D}_{n}(\mathcal{P}) \bigoplus^{-\alpha} \mathcal{H} \mathcal{D}_{n}(\mathcal{P}), \alpha= \pm 1$. For the algebra of Laurent polynomials, the relationship for the dihedral (co)homology and cyclic homology was demonstrated, which is $\mathcal{H} C_{n}\left(\mathcal{P}\left[p, p^{-1}\right]\right) \cong$ $\alpha \mathcal{H} \mathcal{D}_{n}\left(\mathcal{P}\left[p, p^{-1}\right]\right) \bigoplus^{-\alpha} \mathcal{H} \mathcal{D}_{n}\left(\mathcal{P}\left[p, p^{-1}\right]\right), \alpha= \pm 1$. The Morita of dihedral homology was studied. The trace and inclusion maps of the dihedral homology were indicated as follows: tr $:{ }^{\alpha} \mathcal{H} \mathcal{D}_{n}(\mathcal{P} \otimes I) \rightarrow{ }^{\alpha} \mathcal{H} \mathcal{D}_{n}\left(M_{m}(\mathcal{P}) \otimes M_{m}(I)\right) \quad, \quad$ and inc: ${ }^{\alpha} \mathcal{H} \mathcal{D}_{n}\left(M_{m}(\mathcal{P}) \otimes M_{m}(I) \rightarrow{ }^{\alpha} \mathcal{H} \mathcal{D}_{n}(\mathcal{P} \otimes I)\right.$ respectively.

From the sequence $0 \rightarrow \mathcal{P} \rightarrow \mathcal{P}^{\prime} \rightarrow \mathcal{P}^{\prime \prime} \rightarrow 0$, the exact long sequence of the dihedral homology and hyperhomology of polynomial algebra were obtained as follows:

$$
\begin{gathered}
\cdots \rightarrow \mathcal{H} \mathcal{D}_{n}(\mathcal{P}) \rightarrow \mathcal{H} \mathcal{D}_{n}\left(\mathcal{P}^{\prime}\right) \rightarrow \mathcal{H} \mathcal{D}_{n}\left(\mathcal{P}^{\prime \prime}\right) \rightarrow \\
\mathcal{H} \mathcal{D}_{n-1}(\mathcal{P}) \rightarrow \mathcal{H} \mathcal{D}_{n-1}\left(\mathcal{P}^{\prime}\right) \rightarrow \mathcal{H} \mathcal{D}_{n-1}\left(\mathcal{P}^{\prime \prime}\right) \rightarrow \cdots \\
\cdots \rightarrow \mathcal{H} \mathcal{R}_{n}(\mathcal{P}) \rightarrow \mathcal{H} \mathcal{R}_{n}\left(\mathcal{P}^{\prime}\right) \rightarrow \mathcal{H} \mathcal{R}_{n}\left(\mathcal{P}^{\prime \prime}\right) \rightarrow \\
\mathcal{H} \mathcal{R}_{n-1}(\mathcal{P}) \rightarrow \mathcal{H} \mathcal{R}_{n-1}\left(\mathcal{P}^{\prime}\right) \rightarrow \mathcal{H} \mathcal{R}_{n-1}\left(\mathcal{P}^{\prime \prime}\right) \rightarrow \\
\cdots, \text { respectively. }
\end{gathered}
$$

We can apply this result with ([22], [23]) and give use more generalizations.

\section{Acknowledgements}

The authors thank the officials for their help and ideas with the principle draft of the current work.

\section{Declarations}

\section{Funding}

No funding was received.

\section{Contributions}

The authors completed all aspects of the study through diligent work and an analysis of numerous sources and achievements in the field of mathematics. The final manuscript has been read and accepted by the authors.

\section{Ethics Approval}

Not applicable.

\section{REFERENCES}

[1] Hochschild G., "On the cohomology groups of an associative algebra," Annals of Mathematics, Second Series Second Series, Vol. 46, No. 1, pp. 58-67 (10 pages), Jan. 1945. Doi:10.2307/1969145, ISSN 0003-486X, JSTOR 1969145, MR 0011076.

[2] Cartan H. \& Eilenberg S., "Homological algebra," Princeton Mathematical, Princeton University Press, Vol. 19, 1956. Doi.org/10.1515/9781400883844, Isbn 978-0-691 04991-5, MR 0077480.

[3] Tsygan, B. L., “The homology of matrix Lie algebras over rings and the Hochschild homology," Russian Mathematical Surveys, vol. 38, No.2, pp. 198-199, 1983. Doi.org/10.1070/RM1983v038n02ABEH003481.

[4] Connes A., "Non-commutative differential geometry," Publications Mathématiques de L'Institut des Hautes Scientifiques, vol. 62, No.1, pp. 41-144, 1985. Doi.org/10.1007/BF02698807.

[5] Tsygan, B.L. "Homologies of some matrix Lie superalgebras," Funktsional'nyi Analiz i ego Prilozheniya, vol. 20, No.2, pp. 90-91, 1986. Doi.org/10.1007/BF01077286.

[6] Gouda Y. Gh. \& Alaa H. N., "On the trivial and nontrivial cohomology with inner symmetry groups of some classes of operator algebras,” Int. Journal of Math. Analysis, Vol. 3, No.8, pp. 377-384, 2009.

[7] Kadison R. V. \& Ringrose J. R., "Cohomology of operator algebras,” Acta Mathematica, Vol. 126, No., pp. 227-243, 1972.

[8] Gouda Y. Gh., "On the (co)homology theory of index category," Journal of the Egyptian Mathematical Society, vol. 19, No. 3, pp. 137-141, 2011. Doi.org/10.1016/j.joems.2011.12.002.

[9] Helemskii, A. Ya., "Banach Cyclic (Co)Homology and the Connes-Tzygan Exact Sequence,” Journal of the London Mathematical Society, vol. s2-46, No. 3, pp. 449-462, (1992). Doi.org/10.1112/jlms/s2-46.3.449.

[10] Helemskii A. Ya., "Banach cyclic (co)homology as Banach derived functors,” Algebra i Analiz, vol. 3, No. 5, pp. 213-228, 1991. https://zbmath.org/?q=an:0895.46036.

[11] Białkowski, J., Erdmann, K., \& Skowroński, A., "Hochschild cohomology for periodic algebras of polynomial growth,” Journal of Pure and Applied Algebra, vol. 223, No. 4, pp. 1548-1589, 2019. Doi.org/10.1016/j.jpaa.2018.06.018

[12] Quillen D., "Projective modules over polynomial rings," Inventiones mathematicae, vol. 36, No. 1, pp. 167-171, 1976. Doi.org/10.1007/BF01390008.

[13] Suslin A., "Projective modules over polynomial rings are free,” Doklady - Akademiya Nauk SSSR, Earth Science Sections, vol. 229, pp. 1063-1066, 1976.

[14] Penner R., “Long exact sequences of k-groups,” Lecture Notes in Mathematics, Springer, vol. 2262, 2020. Doi.org/10.1007/978-3-030-43996-5_36.

[15] Alaa H. N. Mohamed \& Mohsen A. H. Eid, "Study of the 
homology theory of fuzzy algebra,” International Journal of Research, Vol. 6, No. 3, e-ISSN: 2348-6848, p-ISSN: 2348-795X, 2019.https://journals.pen2print.org/index.php/ ijr

[16] Alaa Hassan Noreldeen Mohamed, “On the cohomology of relative Banach algebras”, Modern Applied Science; Vol. 13, No. 10, ISSN 1913-1844 E-ISSN 1913-1852, 2019. Doi:10.5539/mas. v13n10p1.

[17] Alaa H. N. Mohamed, "Study of the d-infinity differential module,” International Journal of Mathematical Analysis, Vol. 13, No. 10, pp. 487-494, 2019. Doi.org/10.12988/ijma.2019.9849.

[18] Alaa Hassan Noreldeen and Abo-Quota S. A., "Operations on the dihedral homology theory," Applied Mathematical Sciences, Vol. 13, No. 20, pp. 983-990, 2019. https://doi.org/10.12988/ams.2019.98122.

[19] Alaa Hassan Noreldeen, "Treatment of operator algebras through cohomology theory,” Information Sciences Letters,
Vol. 9, No. 1, pp. 1-5, 2020. Doi.org/10.18576/isl/090101

[20] Alaa Hassan Noreldeen Mohamed, "Perturbation differential a-infinity algebra," Applied Mathematics \& Information Sciences, vol. 14, No. 3, pp. 1-5 2020. Doi.org/10.18576/amis/140311.

[21] Alaa Hassan Noreldeen, "Differential graded algebras and

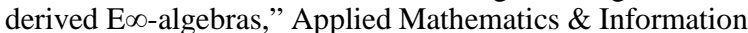
Sciences, vol. 14, No. 4, pp. 673-678, 2020. doi:10.18576/amis/140415.

[22] Min, Jasmine Lee Jia, and Syafrina Abdul Halim. "Rainfall Modelling using Generalized Extreme Value Distribution with Cyclic Covariate," Mathematics and Statistics, Vol. 8, No. 6, pp. $762 \quad-\quad 772,2020$. DOI: $10.13189 / \mathrm{ms} .2020 .080617$

[23] Anton Epifanov, "Analysis of the Element's Arrangement Structures in Discrete Numerical Sequences," Mathematics and Statistics, Vol. 8, No. 2, pp. 173 - 180, 2020 DOI:10.13189/ms.2020.080213 